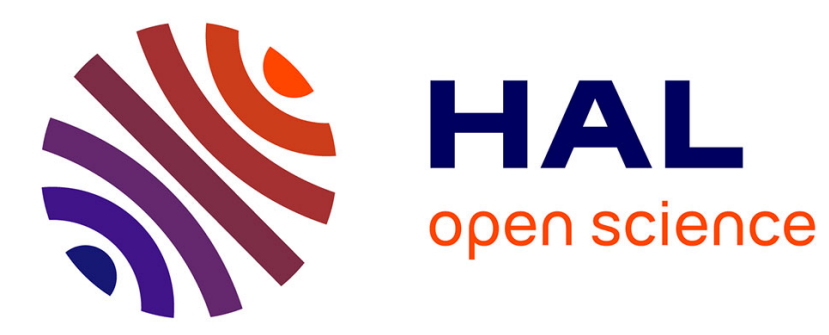

\title{
On the Josephson effect
}

\author{
A. Libchaber
}

\section{To cite this version:}

A. Libchaber. On the Josephson effect. Revue de Physique Appliquée, 1974, 9 (1), pp.1-3. 10.1051/rphysap:01974009010100 . jpa-00243740

\section{HAL Id: jpa-00243740 https://hal.science/jpa-00243740}

Submitted on 1 Jan 1974

HAL is a multi-disciplinary open access archive for the deposit and dissemination of scientific research documents, whether they are published or not. The documents may come from teaching and research institutions in France or abroad, or from public or private research centers.
L'archive ouverte pluridisciplinaire HAL, est destinée au dépôt et à la diffusion de documents scientifiques de niveau recherche, publiés ou non, émanant des établissements d'enseignement et de recherche français ou étrangers, des laboratoires publics ou privés. 


\title{
ON THE JOSEPHSON EFFECT
}

\author{
A. LIBCHABER \\ Groupe de Physique des Solides $(*)$ de l'Ecole Normale Supérieure, \\ 24, rue Lhomond, 75231 Paris $5 \mathrm{e}$, France
}

\begin{abstract}
Résumé. - On présente ici des observations sur l'effet Josephson où la situation théorique est peu claire et l'état des expériences encore peu satisfaisant. Ce sont : 1 . l'apparition d'un nouveau terme dissipatif en $\cos \varphi$ où $\varphi$ est la différence de phase entre les deux supraconducteurs, 2 . la réponse d'une diode Josephson à des fréquences voisines du "gap " (singularité de RiedelWerthamer), 3. l'effet d'une irradiation hyperfréquence sur le courant critique dans les ponts du type Dayem.
\end{abstract}

Abstract. - We discuss topics related to Josephson effects where the theoretical situation is complicated, and experiments still not in a satisfactory stage. These are $: 1$. the appearance of a new dissipative $\cos \varphi$ term, where $\varphi$ is the phase difference between the two supraconductors, 2. the microwave response of a Josephson diode at photon energies near the energy gap, 3. the effect of microwaves on critical current in the so-called Dayem bridges.

The Josephson effect is now more than ten years old [1] and this conference on "detection and emission of electromagnetic radiation with Josephson junctions " indicates well that many applications of the effect are actively studied. The basic physics of the effect are well known now, using a two fluid model, with a pair superconducting current and a quasiparticle normal current. These two currents are not interacting as in the simple two fluid model.

Consider two identical superconductors separated by a thin dielectric layer of thickness $l$ lying in the $\mathrm{XY}$ plane. Josephson description of the barrier is based on a two dimensional field $\varphi(x, y, t)$ representing the increase in the phase of the order parameter $\psi$ on crossing the barrier. $\psi$ is the order parameter introduced by Ginzburg and Landau whose square is proportional to the number of superconducting electron pairs.

The derivatives of $\varphi$ are related to the electromagnetic fields $E$ and $H$ in the junction :

$$
\begin{aligned}
& \frac{\partial \varphi}{\partial t}=\frac{2 \mathrm{e} l E}{h}=\frac{2 \mathrm{e} V}{h} \\
& \nabla \varphi=\frac{2 \mathrm{e} d}{\hbar c} \mathbf{H} \times \mathbf{Z}
\end{aligned}
$$

where $V$ is the voltage across the dielectric and $d=2 \lambda+l, \lambda$ the magnetic field penetration depth. The phase $\varphi$ determines also the supercurrent flowing across the barrier and the total current is :

$$
j=j_{1} \sin \varphi+\sigma_{0}(V) V .
$$

(*) Laboratoire associé au Centre National de la Recherche Scientifique.
The first term represents the supercurrent, the second is the quasiparticle current.

These equations are sufficient to explain the main phenomena predicted by Josephson and later observed.

Another useful presentation has been given by Aslamazov and Larkin [2]. In their model they treat the junction as a two phase region where the amplitude of the separate phases is strongly position dependent. Thus the wave function is composed of two terms :

$$
\begin{aligned}
\psi=\psi_{1}+\psi_{2}=\psi_{0}\left\{f(x) \exp \left(i \varphi_{1}\right)+\right. \\
\left.+[1-f(x)] \exp \left(i \varphi_{2}\right)\right\}
\end{aligned}
$$

$f(x)$ goes to unity in superconductor 1 and 0 in superconductor $2, \psi_{0}$ is the amplitude far from the junction. Defining the current in terms of a gradient operator, the current is proportional to $\psi_{0}^{2} f \nabla f \sin \left(\varphi_{1}-\varphi_{2}\right) \quad$ for the supercurrent and $\nabla f \sigma V$ for the quasiparticle current. Aslamazov and Larkin's representation is very useful in the case of a resistively shunted junction.

Recently Notarys, Yu and Mercereau [3] have generalized this treatment, in the case of a high current density junction, by allowing the phase to be also a function of position $\varphi=\varphi(x)$. In the supercurrent a new $\cos \left(\varphi_{1}-\varphi_{2}\right)$ term appears and:

$$
\begin{aligned}
j_{\mathrm{s}} \propto & \psi_{0}^{2} f \nabla f \sin \left(\varphi_{1}-\varphi_{2}\right)+ \\
& +\psi_{0}^{2} f^{2}\left(\nabla \varphi_{1}+\nabla \varphi_{2}\right)\left[1+\cos \left(\varphi_{1}-\varphi_{2}\right)\right] .
\end{aligned}
$$

This new supercurrent term is important for a high current density junction.

This summarizes the two useful models in the classical regime. We will now present cases where the 
situation is more complicated, and the experiments in a still not satisfactory stage.

1. The dissipative $\cos \varphi$ term [4]. - In the two fluids model, the losses are associated with the quasiparticle current $\sigma_{0}(V) V$ (eq. (3)).

Josephson [5] predicted that a cross term should be added to the current, a quasiparticle-pair interference current of the form :

$$
j_{\mathrm{q}, \mathrm{p}}=\sigma_{1}(V) V \cos \Phi
$$

which is a breakdown of the two fluids model. This term would thus participate to the losses.

The first experimental observation of this term comes from an experiment by Pedersen, Finnegan and Langenberg [4], on the linewidth of the Josephson plasma resonance. They could explain the dependence of the $Q$ of the plasma resonance with magnetic field only by introducing a $\cos \Phi$ dependent loss term. The sign of $\sigma_{1}(V)$ had to be negative. This surprising result has not yet been confirmed by other experiments. The physical origin of this new term is also not very clear, and we refer to Professor Langenberg's paper at this conference for a detailed presentation.

2. The high frequency response and the Riedel [6] Werthamer [7] singularity. - The high frequency response of a Josephson junction to high frequency radiation, $h v>2 \Delta$, is an important problem. Exposed to microwave radiation, a Josephson tunnel junction will exhibit two types of current steps re ated to the supercurrent and the quasiparticle current. The supercurrent will exhibit steps for voltages $2 \mathrm{e} V=n h v$, and they are described by the equation :

$$
I_{\mathrm{dc}}(V)=I_{\mathrm{J}}(0) \sum_{n=0}^{\infty}\left|J_{n}(2 \alpha)\right| . \delta\left(V \pm \frac{n h v}{2 \mathrm{e}}\right)
$$

where

$$
\alpha=\frac{\mathrm{e} V_{\mathrm{rf}}}{h v}
$$

The quasiparticle current will also exhibit steps, image of the gap, for voltages such as :

$$
2 \Delta \pm n h v=\mathrm{e} V \text {. }
$$

The plus sign corresponds to stimulated emission of photons, and the minus sign to photon-assisted tunneling.

Riedel [6] and Werthamer [7] have shown that the amplitude of the Josephson current displays a logarithmic singularity, related to the singularity of the BSC quasiparticle density of states at the edge of the superconducting energy gap. Thus the Josephson radiation-induced steps will show an amplitude singularity when the step lies at the gap voltage $2 \Delta / \mathrm{e}$. If we shine radiation at a frequency such that the first Josephson step falls at the gap, its amplitude will thus be very large. But the third, fifth... steps will fall at the gap induced by stimulated emission of photons from quasiparticles, and will thus also resonate (the charge of a pair is $2 \mathrm{e}$, and for a quasiparticle e). This divergence is shown on figure 1 . There we shine microwave radiation of wavelength $\lambda=620 \mu$ from a carcinotron tube on a $\mathrm{Ta} / \mathrm{Sn}$ point contact junction [8]. One sees clearly that the amplitude of the odd steps is larger than that of the even one. The Riedel peak has been studied by Hamilton and Shapiro [9], and Buckner, Finnegan and Langenberg [10] : the singularity is clearly rounded off; from the data it seems that gap anisotropy is responsable for that, but the analysis is far from complete. Also, results for frequency much larger than the gap are very puzzling, giving steps of amplitude much larger than the simple theory predicts. It would be useful to have a theory of the infrared response for a resistively shunted junction, combining Aslamazov and Larkin's model with a Werthamer type analysis, coupled with precise data.

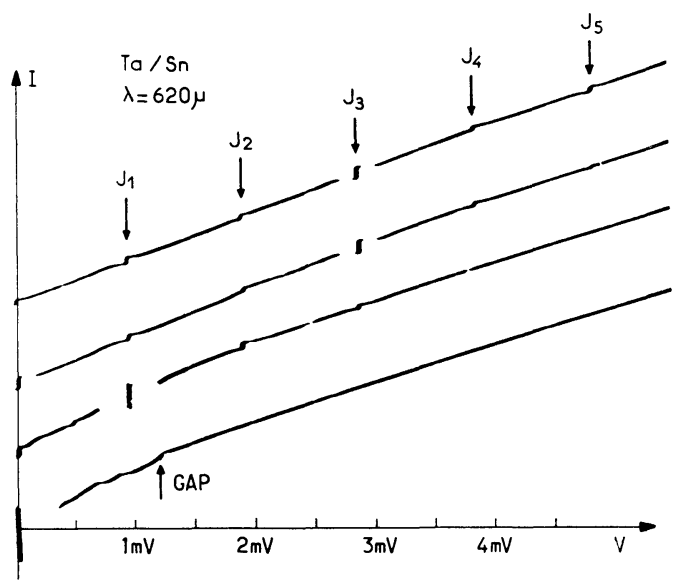

FIG. 1. - I- $V$ characteristic of a Ta/Sn superconducting point contact at $T=1.2 \mathrm{~K}$. The various curves correspond to increasing amplitude of the microwave power from a carcinotron tube, at a wavelength $\lambda=620 \mu$.

3. The Dayem bridge. - The Dayem bridge represents a type of Josephson junction different from the tunnel junction. It consists of a superconductor constriction-superconductor film. The constriction is typically of the order of one micron. Far from $T_{\mathrm{c}}$ the model given for such a junction [11] was that at the critical current, vortices start to move across the junction at the frequency $2 \mathrm{e} V=n h v$. This supposes that $\xi$, the coherence length, is smaller than the constriction width. Close to $T_{\mathrm{c}}$ when $\xi$ becomes larger, another model has been developed by Rieger, Scalapino and Mercereau [12]. One is faced there with a one-dimensional superconductor. To describe the time evolution of the system, they consider this state as being in constant acceleration. Thus one has free particle acceleration until the current $j$ reaches the critical velocity. When the critical velocity is reached on a time $\tau \sim h / 2 \mathrm{eV}$, the amplitude of the super- 
conducting state becomes unstable, decays towards zero on a time scale comparable to the pairing time $\tau=h / 2 \Delta$, and the process starts again. The result is a time-dependent state of superconductivity at finite voltage oscillating at the Josephson frequency. It is a time-dependent two-fluid model. When the voltage is close to the gap, it is not clear whether the ac current will simply follow the Josephson frequency, and experimental results would be very useful there.

Such a junction, close to $T_{\mathrm{c}}$, presents a peculiar anomaly, observed first by Dayem and Wiegand, when subject to microwave irradiation. The dc current, instead of following a simple $J_{0}\left(\mathrm{e} V_{\text {rf }} / h v\right)$ Bessel law, first increases strongly with an increase of $V_{\text {rf }}$. This effect never received a very satisfactory explanation. Very recently, Notarys, Yu and Mercereau [3] gave an explanation based on the fact that it is a high current density system. Using eq. (5), they showed that the $\mathrm{rf}$ enhancement of the dc supercurrent is due to the new $\psi_{0}^{2} f^{2} \nabla \varphi\left|1+\cos \left(\varphi_{2}-\varphi_{1}\right)\right|$ term that they have introduced. This term becomes significant in junctions where the dimension parallel to the current flow approximated the de Broglie wavelength of the current. It would be important to confirm if this model is sufficient to explain the rf enhancement of the dc supercurrent in all bridges.

\section{References}

[1] Josephison, B. D., Phys. Lett. 1 (1962) 251.

[2] Aslamazov, L. G., Larkin, A. I., JETP Lett. 9 (1969) 87.

[3] Notarys, H. A., Yu, M. L., Mercereau, J. E., Phys. Rev. Lett. 30 (1973) 743.

[4] Pedersen, N. F., Finnegan, T. F., Langenberg, D. N., Phys. Rev. B 6 (1972) 4151.

[5] Josephson, B. D., Adv. Phys. 14 (1965) 419.

[6] Riedel, E., Z. Naturforsch. 19a (1964) 1634.

[7] Werthamer, N. R., Phys. Rev. 147 (1966) 255.
[8] Maurer, J., Libchaber, A., to be published.

[9] Hamilton, C. A., Shapiro, S., Phys. Rev. Lett. 26 (1971) 426.

[10] Buckner, S. A., Finnegan, T. F., Langenberg, D. N., Phys. Rev. Lett. 28 (1972) 150.

[11] Anderson, P. W., Dayem, A. H., Phys. Rev. Lett. 13 (1964) 195.

Wyatt, A. F. G., Dmitriev, V. M., Moore, S. W., Sheard, F. W., Phys. Rev. Lett. 16 (1966) 1166.

[12] Rieger, T. J., Scalapino, D. J., Mercereau, J. E., Phys. Rev. B 6 (1972) 1734. 